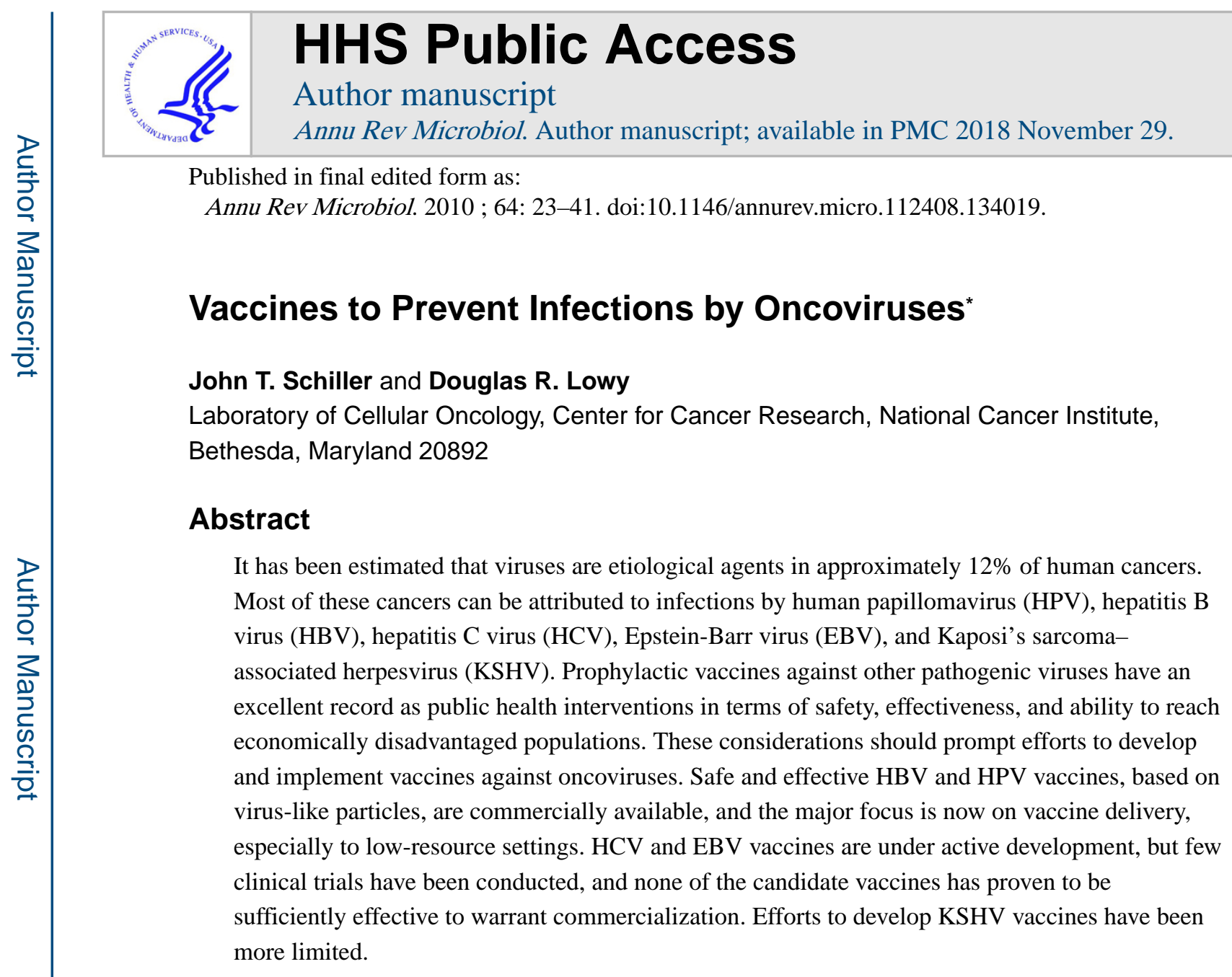

Keywords

HBV; HPV; HCV; KSHV; EBV; HTLV

\title{
INTRODUCTION
}

Viral infections cause a substantial proportion of human cancers. It is estimated that viruses are etiologic agents for approximately $12 \%$ of all human cancers worldwide (63). Human papillomavirus (HPV), hepatitis B virus (HBV), hepatitis C virus (HCV), Epstein-Barr virus (EBV), Kaposi's sarcoma-associated herpesvirus (KSHV), human immunodeficiency virus (HIV), and human T cell lymphoma virus (HTLV) are well-established contributors to human cancer. The percent of worldwide cancers caused by the individual viruses ranges from $5.2 \%$ for HPV to $0.03 \%$ for HTLV (Table 1). These viruses induce many different types of cancers, and the fraction of a particular cancer attributable to a specific virus varies greatly (Table 1). Nonviral infectious agents also cause a significant fraction of human cancers. Most notably, the bacterium Helicobacter pylori is an etiological agent in

\footnotetext{
${ }^{*}$ This is a work of the U.S. Government and is not subject to copyright protection in the United States.

The authors are inventors on U.S. Government-owned patents covering the papillomavirus VLP vaccines that have been licensed to Merck and GlaxoSmithKline.
} 
approximately $5 \%$ of human malignancies, mostly gastric cancers. However, this review focuses exclusively on the viruses most commonly implicated in cancers.

The determination that a cancer has a viral etiology provides an opportunity for cancer prevention by vaccination to prevent or treat the viral infection that induces the cancer. Prophylactic vaccines against viral infections, such as smallpox, polio, and measles, have been among the most successful public health interventions ever devised, so this strategy is in principle an attractive one for reducing the incidence of infections by cancer-causing viruses. This review of oncoviruses and the development of vaccines to combat them examines the factors that have enabled this strategy to be successfully employed against two oncoviruses, HBV and HPV. It also addresses issues related to the feasibility, potential public health impact, and commercial prospects in extending this approach to some other oncoviruses. HIV vaccines are not addressed here because the extensive literature on this topic has been the subject of numerous recent reviews $(19,76)$. The emphasis is on prophylactic vaccines and animal models for testing them, since successful vaccines against viral infections, including HBV and HPV, are mostly preventive. Current prophylactic vaccines are thought to function primarily through the induction of virus-neutralizing antibodies.

Therapeutic vaccines have been less successful, and there is no licensed therapeutic vaccine against an oncovirus. However, persistent oncovirus infections, and the premalignant lesions they induce, are attractive candidates for therapeutic vaccines, particularly if the virally induced lesions selectively retain expression of viral oncogenes (41). Continuing advances in our basic understanding of the induction and regulation of T cell- and other cell-mediated immune responses, coupled with the development of increasingly sophisticated vaccination strategies, increase the likelihood that more effective therapeutic vaccines against oncoviruses will be forthcoming.

In general, vaccination programs have been exceptionally effective in overcoming health care disparities based on socioeconomic status. This is an important consideration because most cases of virus-associated cancers occur in developing countries. It has been estimated that $26 \%$ of cancers in developing countries are caused by an infectious agent, compared with $8 \%$ of cancers in developed countries (63). Antiviral vaccines may represent one of the best opportunities for reducing cancer rates in low-resource settings. However, because it often takes decades for a primary viral infection to progress to cancer, the return on investments in vaccination programs to prevent oncovirus infections will be substantially delayed. Induction of significant disease during primary infection, in addition to cancer, can increase the attractiveness of an oncovirus as a target for vaccine development and deployment.

\section{HEPATITIS B VIRUS}

The development and implementation of the hepatitis B virus (HBV) prophylactic vaccine for the prevention of hepatocellular carcinoma (HCC) has served in many ways as the model for the development of other oncovirus vaccines. It was the first vaccine generated by recombinant DNA technology, the first oncovirus vaccine to be licensed, and the only 
vaccine that has been shown to decrease the incidence of an oncovirus infection in a general population and to prevent human cancer.

$\mathrm{HBV}$ is a relatively small, 42-nm virus of the Hepadnaviridae family. It is composed of an outer lipid envelope, containing three forms of the S envelope protein, also called HBsAg, surrounding a nucleocapsid. The nucleocapsid is composed of a $3.2-\mathrm{kb}$ partially double DNA genome and a virally encoded polymerase within a capsid composed of the core protein, also called HBcAg. During infection, two additional enveloped viral particles are generated. They are smaller spherical and tubular structures with a diameter of $22 \mathrm{~nm}$. These particles contain only S, the shortest version of HBsAg. The S particles do not contain the viral genome and are therefore noninfectious (53).

The HBV infectious cycle is highly adapted to hepatocytes, with no clear evidence for extrahepatic replication. As with other Hepadnaviridae, replication is species restricted, so there is no small-animal model for HBV replication or vaccination studies, although experimental models involving woodchuck, ground squirrel, and duck Hepadnaviridae have been developed (53). Because the genome replicates though an RNA intermediate, its mutation rate is high (16), more similar to the rate of a typical RNA virus than to the rate of a typical DNA virus.

$\mathrm{HBV}$ is one of the most prevalent blood-borne viruses. Over 350 million people are chronically infected worldwide, and 4.5 million new infections occur each year (32). Infection rates are particularly high in East Asia, sub-Saharan Africa, and the Amazon basin. In areas of high endemicity, transmission is most commonly vertical, from mother to child, or horizontal between children. In areas of low endemicity, the predominant routes of transmission are sexual activity and intravenous drug use or other exposures to contaminated blood (86). Fortunately, serologic and molecular screening of the blood supply has made transfusion-related infection rare in developed countries.

HBV infection causes a wide range of liver disease, from subclinical infection to acute selflimiting hepatitis and fulminant hepatitis. Most available evidence suggests that the virus is not directly cytopathic. Rather, disease is thought to result primarily from cellular immune responses to viral products of infected cells (15). Chronic infection can lead to cirrhosis and eventually to HCC. Approximately 620,000 people die annually from acute and chronic HBV infections (32). There is a strong inverse correlation between age at acquisition and probability of chronic infection (22). Hence, prenatal or perinatal transmission from an infected mother is strongly associated with establishment of a chronic carrier state.

The epidemiologic association of chronic HBV infection and HCC is strong, with an attributable fraction of over $50 \%$ (Table 1). In a prospective study in Taiwan, the relative risk of developing HCC in HBsAg-positive men was over 60 times greater than the risk in HBsAg-negative men (6). In HCC, the viral genome can be maintained as an autonomous replicon, or it can be integrated into host DNA (56).

Development of HBV vaccines in the late 1970s and early 1980s was based on the clear understanding that $\mathrm{HBV}$ was a major cause of viral hepatitis, in addition to its emerging association with cancer. Thus, a primary impetus in developing prophylactic HBV vaccines 
was to prevent acute hepatitis, chronic HBV infection, and cirrhosis. Studies in the 1970s determined that passive transfer of anti-HBsAg immunoglobulin could be used for postexposure prophylaxis against acute and chronic HBV infection (59). These studies provided a compelling rationale for developing prophylactic vaccines to induce HBVneutralizing antibodies. The first vaccines, licensed initially in 1981-1982, were based on purified and inactivated 22-nm HBsAg particles harvested from individuals with chronic HBV infection. Although these vaccines were given to millions of individuals, there were theoretical concerns about the safety of human-blood-derived products (86), providing the impetus for development of the first commercial vaccines based on recombinant DNA technologies. It was discovered that expression of the $\mathrm{S}$ protein alone in yeast (Saccharomyces cerevisiae) led to self-assembly of lipid membrane-containing particles that were morphologically indistinguishable from the 22-nm S particles found in the blood of $\mathrm{HBV}$-infected individuals (60). Purified S particles produced by this technology provided a safe, unlimited, and low-cost source of vaccine. Recombinant yeast-derived vaccines became commercially available in 1986 . They are now produced by a number of manufacturers in the United States, Europe, India, South Korea, Indonesia, and Cuba for well under a dollar per dose (58). Several hundred million doses of these recombinant vaccines have been administered worldwide, and their safety record has been excellent.

A standard full vaccination course involves three intramuscular injections at 0,1 , and 6 months. The WHO recommends that all infants be vaccinated, with the first dose administered within $24 \mathrm{hr}$ of birth. This schedule generates protective levels of $\mathrm{HBsAg}$ antibodies in more than $95 \%$ of healthy infants and adults. The decline from peak antibody titers is biphasic, with a relatively rapid decline in the first one to two years, followed by a slow rate of decline thereafter. Remarkably, HBsAg antibodies can be detected in a majority of vaccinees even 20 years after vaccination, and a rapid anamnestic response is seen after boosting, even in individuals without detectable anti-HBsAg (67). The long-term immunological memory induced by the vaccine provides long-term protection from acute and chronic HBV infection. Hence, boosters are not routinely recommended.

The vaccine offers excellent protection against all eight of the recognized HBV genotypes (A to $\mathrm{H}$ ). Because of their high production and mutation rate (16), there has been concern that, in vaccinated populations, there will be strain replacement by variants, with changes in the immunodominant neutralization epitopes of the $S$ protein. While such strains have indeed been documented in some vaccinees, to date they do not appear to pose a major threat to the overall effectiveness of the vaccination programs. Perhaps these variants are generally less fit than the vaccine-sensitive strains.

Vaccination programs in industrialized countries initially focused on high-risk groups such as infants of infected mothers, health care workers, and drug users. However, coverage generally remained low in these groups. In addition, a substantial minority of HBV-infected individuals lacked all the major risk factors. This situation led to the initiation of universal vaccination programs, even in many countries with relatively low infection rates, and consequently to a rapid increase in coverage. According to WHO estimates, the percent of infants vaccinated worldwide increased from $1 \%$ in 1990, to $30 \%$ in 2000 , to $60 \%$ in 2006. Two major factors contributed to this dramatic increase. One was the availability of low-cost 
vaccine from emerging country manufacturers. The second was support for vaccination programs in the world's poorest nations by the Global Alliance for Vaccines and Immunization (GAVI) and the Vaccine Fund.

Universal HBV vaccination has had a substantial impact on the rates of acute HBV-related hepatitis and on carrier rates in countries with well-established vaccination programs. The prevalence of childhood HBsAg has fallen from $9.8 \%$ to $0.7 \%$ in Taiwan and from $10 \%$ to $0.6 \%$ in the Gambia (86). A reduction in childhood HCC has also been documented in Taiwan, which adopted universal infant vaccination in 1984. In a landmark study, the annual incidence of HCC in 6- to 14-year-old children decreased from 0.7/100,000 in 1981-1986 to 0.36/100,000 in 1990-1994 (12). A recent follow-up study of 6- to 29-year olds showed a greater than two-thirds reduction in the relative risk of HCC in individuals vaccinated at birth (13). Because chronic infections induced in infancy are largely responsible for adultonset HBV-associated HCC, which generally occurs after age 40, there is optimism that further decreases in the incidence of adult HBV-associated HCC will be observed in the coming decades. The central challenge now is to increase vaccine coverage rates around the world.

\section{HUMAN PAPILLOMAVIRUS}

Human papillomavirus (HPV), which replicates in multilayered epithelia, is the second oncovirus for which a prophylactic vaccine has been developed. Genital HPV infection is the most common sexually transmitted viral disease, with point prevalence rates greater than $25 \%$ for young sexually active individuals. Infection is acquired by many women soon after initiating sexual activity, with a lifetime risk estimated to be at least $80 \%$ (5). Many women have multiple HPV infections. More than $30 \mathrm{HPV}$ types can infect the genital tract, and about 15 of them appear to have oncogenic potential (7). The vast majority of infections, whether by an oncogenic or nononcogenic HPV type, are self-limited, typically clearing 612 months after their onset, presumably by cell-mediated immune mechanisms (5).

However, persistent cervical infection, which occurs in less than $10 \%$ of infections, places the individual at increased risk of developing precancer and invasive cancer. The interval between infection and the development of invasive cancer usually takes at least 20 years, although it may sometimes be substantially shorter (7).

Virtually all cases of cervical cancer are attributable to infection by an oncogenic HPV type (8). Worldwide, cervical cancer is the second most common cause of death from cancer in women. About $80 \%$ of these malignancies occur in the developing world, largely because, in contrast to developed countries, effective cervical cancer-screening programs have not been widely implemented in low-resource settings (63). HPV infection can also cause other anogenital epithelial malignancies, including some vulvar, vaginal, anal, and penile cancers, as well as a substantial proportion of oropharyngeal cancers (31). HPV16, initially isolated in 1983 (20), is most often detected in cancers, accounting for about $50 \%$ of cervical cancers and about $90 \%$ of the noncervical HPV-associated cancers. HPV18 accounts for about $20 \%$ of cervical cancers and $5 \%$ of the noncervical cancers. 
Papillomaviruses are nonenveloped viruses whose $\sim 8$-kb circular DNA genome encodes several nonstructural viral proteins (37). Two genes encode the structural viral proteins, L1 and L2. L1 contains the immunodominant neutralization epitopes. The current commercial HPV vaccines are noninfectious subunit vaccines composed of L1 virus-like particles (VLPs) (71). L1 protein expression in eukaryotic cells is sufficient for the efficient selfassembly into VLPs composed of $72 \mathrm{~L} 1$ pentamers. When used for systemic immunization, they induce high titers of serum-neutralizing antibodies to conformation-dependent L1 epitopes (45). The immunodominant L1 neutralization epitopes are predominantly genotype specific.

Although animal papillomavirus studies showed L1 VLPs could induce strong protection against high-dose homologous viral challenge, it was unclear whether such protection would extend to people. First, HPVs do not infect other species, and none of the experimental papillomavirus models involved genital tract infection, although two were oral-mucosal models. Second, cervical HPV infection is a local mucosal infection, and it was unknown whether a systemically administered subunit vaccine, which effectively induces systemic $\mathrm{IgG}$, but not secretory IgA, would be effective. Third, the track record of human clinical trials with vaccines against local sexually transmitted infections, such as HSV2, has been disappointing.

Because of the importance of cervical cancer, women were the main group for testing HPV vaccine efficacy. The first proof-of-principle results, which tested a monovalent HPV16 L1 VLP vaccine manufactured by Merck, indicated that the vaccine was about $80 \%$ effective in preventing transient infection with HPV16 and, remarkably, 100\% effective in preventing persistent infection by this type (49). However, there was no protection against cervical dysplasia not associated with HPV16 infection, confirming that vaccine efficacy would be type restricted. These encouraging results were followed by the development and phase III testing of two commercial vaccines manufactured by Merck and GlaxoSmithKline (GSK) (71). The Merck vaccine is a quadrivalent VLP vaccine with a simple aluminum salt adjuvant. It contains VLPs from HPV16 and HPV18, which together account for about 70\% of cervical cancer, as well as VLPs from HPV6 and HPV11, which together account for about $90 \%$ of external genital warts. The GSK vaccine is a bivalent vaccine composed of VLPs from HPV16 and HPV18 and AS04, the same proprietary adjuvant used in GSK's HBV vaccine. Both HPV vaccines are delivered in three intramuscular doses over a span of six months. The clinical trial results from both vaccines have confirmed their high efficacy against infection and neoplasia caused by the HPV types targeted by the respective vaccine $(46,62)$. The FDA mandated that, prior to licensure, the vaccine demonstrate it could prevent moderate or severe cervical intraepithelial neoplasia (CIN2+), as this degree of dysplasia triggers treatment in the United States. As standard of care requires intervention for CIN2+, it would have been unethical to use cervical cancer as an end point. Both vaccines showed at least $98 \%$ protection against CIN2+ attributable to HPV16 or HPV18. In addition, the Merck vaccine showed a similar high degree of protection against moderate and severe vulvar and vaginal dysplasia and external genital warts. The Merck vaccine also provides about $90 \%$ protection against external genital warts in males. Thus far, protection from the vaccines has remained strong, more than 5 years by the Merck vaccine and more 
than 6 years by the GSK vaccine. Models based on the durability of antibody responses suggest that protection will last substantially longer (17).

Some cross-protection against nonvaccine types has been seen with each vaccine. The Merck vaccine showed partial protection (46\%) against persistent cervical infection with HPV31, which is phylogenetically related to HPV16 (10). The GSK vaccine showed partial protection against HPV31 (67\%), HPV33 (42\%), also related to HPV16, and HPV45 (71\%), which is related to HPV18 (62). The differences in cross-protection may be related to subtle differences in the display of L1 epitopes, the AS04 adjuvant in the GSK vaccine, or its greater immunogenicity.

The FDA licensed the Merck vaccine in 2006 and the GSK vaccine in 2009 for young females (9-26 for the Merck vaccine and 10-25 for the GSK vaccine). The CDC Advisory Committee on Immunization Practices (ACIP) recommends routine vaccination for the target group of 11- to 12-year-old girls (because of the importance of immunization prior to initiating sexual activity) and catch-up vaccination for the older girls/women (73). The Merck vaccine was also approved in 2009 for 9- to 26-year-old males, and the ACIP gave a permissive recommendation for its use in the approved male population. Transmission models of HPV infection suggest that high coverage of one gender should be sufficient to reduce the prevalence of the vaccine types in both genders. As women bear the greater risk of serious HPV infection, it is reasonable to give more emphasis to female vaccination than to male vaccination.

A unique aspect of the HPV life cycle probably contributes to the high efficacy of the VLP vaccines against mucosal and cutaneous infection. Studies in a mouse cervicovaginal challenge model indicate that HPV infection requires disruption of epithelial integrity (69). The first infectious steps take place on the basement membrane that separates the dermis from the epithelium, with subsequent transfer of the virus to the target epithelial cell. It is likely that the microtrauma associated with infection leads, in VLP-immunized animals, to exudation of systemic neutralizing antibodies at potential sites of infection. In the model, passive transfer of immune serum also protects, implying that most protection is attributable to neutralizing antibodies.

Many industrialized countries have incorporated the current HPV vaccines into their national vaccine programs $(52,73)$. However, the vaccines have two important limitations for the developed world. First, they do not appear to have activity against prevalent infection. It is therefore important that they be administered prior to the initiation of sexual activity to maximize their cost-effectiveness. Second, their type-restricted protection means that they will not prevent $25-30 \%$ of serious HPV infections. They will have even less impact on overall genital infection rates, because the vaccine-targeted types induce only a minority of these infections (5). For the developing world, high vaccine cost, together with the need for three injections, makes it less likely that the vaccines will be widely implemented in locations where they might have the greatest public health impact.

As young adolescents tend to mount a stronger immune response than older teenagers, protective immunity might be possible for many individuals with only two immunizations. 
Alternatively, L1 could also be incorporated into a live virus vaccine, such as an adenoassociated virus, which might induce immunity with only one dose. Incorporating L1 into an existing vaccine could drastically reduce cost and perhaps reduce the number of doses. This approach has been shown to be feasible in preclinical studies with HPV16 L1 expressed by a Salmonella typhi vaccine strain used to protect against typhoid fever or by a measles virus vaccine strain $(11,28)$. The availability of a high-throughput pseudovirus-based in vitro neutralization assay should facilitate second-generation vaccine development (72).

For the developed world, the integration of vaccination and existing cervical cancer screening programs must be carefully considered (55). Increasing the protection to additional HPV types could, in principle, drastically reduce the cost of screening programs, in conjunction with lowering the incidence of potentially serious HPV infections. For the current vaccine manufacturers, it is reasonable to achieve this goal by increasing the valency of the vaccine by adding VLPs from additional oncogenic HPV types. Merck has indicated they are testing a non-avalent vaccine that, if its protective activity is similar to that of the current vaccine, could theoretically protect against $90 \%$ of potentially oncogenic infections (7). An alternative approach is to develop a vaccine based on the presence in the L2 minor capsid protein of subdominant, but broadly cross-neutralizing, epitopes. Such epitopes are usually poor choices as immunogens because, in most viruses, epitopes of this class tend to be transiently exposed after the virus has bound the cell surface. However, HPV is unusual, as the L2 epitopes are exposed for hours while the virus is on the basement membrane, before transfer to the epithelial cell (44). L2 polypeptides can be produced in bacteria, which should make it relatively inexpensive to manufacture them. Although L2 is less immunogenic than L1 VLPs, this approach has been protective in preclinical models, and an improved L2 immunogen that is a fusion protein composed of L2 polypeptides from several HPV types has recently been described (38). Such L2 immunogens, if successful, have the potential to prevent nongenital HPV infection in addition to genital HPV infection.

\section{HEPATITIS C VIRUS}

Infection by hepatitis C virus (HCV) was first described in 1975 as a case of "non-A, non-B hepatitis" (26). However, because it could not be propagated in cultured cells, the virus was not isolated and characterized until 1989, using recently developed molecular cloning techniques. Unique among oncoviruses, $\mathrm{HCV}$ is an RNA virus with an exclusively cytoplasmic lifestyle. Specifically, it is a member of the Flaviviridae family with a 9600-nt positive-sense RNA genome that is translated into a single polyprotein and then proteolytically processed into a core protein (C), two envelope proteins (E1 and E2), and several nonstructural (NS) proteins (83). HCV only infects humans and chimpanzees, so there is no immunocompetent small-animal model. Replication of HCV in cultured cells has only recently been accomplished, based upon the isolation of a clone with unique in vitro replicative capacity (42).

$\mathrm{HCV}$ is primarily transmitted percutaneously and, to a lesser extent, sexually. The development of diagnostic tests for HCV has greatly diminished transmission through blood or related products, such that transmission is now most often due to unsafe injection practices. Liver hepatocytes are the primary site of replication. Acute infection is often 
asymptomatic, but approximately $70 \%$ of infections become persistent, frequently leading to liver cirrhosis and HCC. More than 170 million people are chronically infected with HCV worldwide, and there are 3-4 million new infections each year (74). Standard treatment of chronic $\mathrm{HCV}$ infection involves a combination of pegylated interferon alpha and ribavirin (a purine nucleoside analog). However, this treatment does not produce a sustained response in approximately $50 \%$ of patients, it is often discontinued due to side effects, and it is too expensive for most infected individuals worldwide. Thus, there is a considerable need for an inexpensive vaccine that could either prevent infection or cure chronic infections.

There is a strong epidemiological association of HCV infection with HCC. A meta-analysis reported an OR for HCV positivity (in HBV negatives) of 17.3. The OR for HCV/HBV double positives was 165 , suggesting a synergistic effect of infection with these two viruses. $\mathrm{HCV}$ is associated with almost 200,000 HCC cases annually, and it is the leading cause of HCC in Europe, Japan, and the United States, with an attributable fraction of $60 \%$ or greater (9). HCC typically develops after decades of infection in a setting of chronic inflammation and cirrhosis. Therefore, as with HBV infection, the chronic damage and repair of hepatocytes seen in persistent $\mathrm{HCV}$ infection likely contributes indirectly to carcinogenesis. HCV RNA is not always present in malignant cells, in contrast to HPV-associated malignancies.

Traditional prophylactic vaccines based on induction of virion-neutralizing antibodies have not been successfully generated against HCV. The lack of an in vitro infection/production system hindered the development of immunogens and assays to assess neutralizing activity. However, retroviral vectors pseudo-typed with $\mathrm{HCV}$ envelope proteins have enabled the development of in vitro neutralization assays (4). Nevertheless, the extreme genetic variability of the virus remains a major impediment to vaccine development (77). Because of its high production and clearance rate ( $10^{12}$ particles per day) and error-prone polymerase, the virus is maintained within a chronically infected individual as a swarm of related clones, much like HIV. Strain-specific neutralizing antibodies directed against the envelope proteins can be detected early in infection, and early clearance is associated with high titers of these antibodies (64). However, in many cases, the initial neutralizing antibody response is weak, and its induction leads to the evolution of escape mutants. More broadly reactive neutralizing antibodies are often generated during chronic infection, but they are unable to clear infection. Nonneutralizing E2 antibodies that could block the binding of crossgenotype neutralizing antibodies to a neighboring epitope were recently detected in HCVinfected individuals (87). These findings raise hopes that an effective prophylactic vaccine might be based on conserved polypeptide immunogens that preferentially induce broadly cross-genotype neutralizing antibodies without concomitant induction of interfering antibodies. Other neutralizing antibody escape mechanisms that might need to be overcome include shielding of neutralizing epitopes by glycosylation, association of the virions with LDL and other serum lipoproteins, and direct cell-to-cell transfer of the virus (77).

Clearance of acute HCV infection is also characterized by a strong and broad Th1-skewed CD4 $\mathrm{T}$ cell response (21). In addition to direct effector mechanisms, CD4 T cells are probably critical for effective neutralizing antibody and CD8 T cell-mediated responses. T helper responses characteristically become progressively weaker and more narrowly focused 
during chronic infection (21). In addition, the T cells that remain typically have maturation and functional defects.

Because acute $\mathrm{HCV}$ infection is usually asymptomatic, the most critical goal for an $\mathrm{HCV}$ vaccine is not to induce sterilizing immunity but rather to prevent or control persistent infection. A number of polypeptide, VLP, naked DNA, and viral-vector vaccine candidates have been developed to target various sets of HCV antigens $(36,77)$. Most have attempted to generate neutralizing antibodies to the envelope proteins and a broad-based $\mathrm{T}$ cell response. In prophylactic trials involving mostly homologous strain challenge of a small number of chimpanzees, several vaccines have demonstrated some protection against chronic, but not acute, infection.

It will likely be easier to induce potent $\mathrm{T}$ cell responses in naïve individuals who have not undergone the profound HCV-induced suppression of their virus-specific helper T cell responses. In addition, the possibility of vaccine-induced immunopathology would likely be greater in persistently infected patients who have already undergone substantial liver damage. Nevertheless, vaccine developers appear to be focused more on therapeutic applications to clear or control chronic infection. The large number of currently infected individuals, the limitations of current therapies, and the relative ease of conducting therapeutic efficacy trials are factors contributing to this emphasis. Several relatively small clinical trials of polypeptide or viral vector-based vaccines in chronically infected patients have been completed (77). All vaccines safely induced immune responses, but virologic responses were limited, at best. There currently appears to be sufficient public and commercial sector support to ensure the continued development and clinical testing of $\mathrm{HCV}$ vaccines. It is likely that many of the future therapeutic efficacy trials will be conducted in conjunction with standard of care interferon-based antiviral therapy.

\section{KAPOSI'S SARCOMA-ASSOCIATED HERPESVIRUS}

Kaposi's sarcoma-associated herpesvirus (KSHV), also called human herpes virus-8 (HHV-8), is a large, 130-kDa DNA gamma-herpesvirus, the same herpesvirus subfamily as EBV (30). As is characteristic for this subfamily, KSHV predominately infects lymphoblastoid cells. It has the two-phase life cycle common to all herpesviruses: a productive lytic phase, in which large numbers and amounts of the viral gene products are produced, and a latent phase, in which the genome is maintained as a low-copy replicon, with low expression of a limited number of viral genes. Infection rates for KSHV are often lower and more varied geographically than for EBV and many other herpesviruses. Seroprevalence rates range from less than $5 \%$ in the United States and northern Europe to more than 50\% in some Mediterranean and African countries (30). KSHV appears to be transmitted primarily through saliva, although vertical, sexual, blood, and transplant-related transmission are also possible (65).

KSHV was first identified in 1994 via a molecular approach involving differential display of DNA fragments comparing normal and tumor tissue of a case of AIDS-associated Kaposi's sarcoma (KS) (14). KS is an unusual, often multifocal, tumor characterized by the presence of spindle-shaped cells of uncertain origin and extensive angiogenesis and inflammatory cell 
infiltrates. In contrast to most malignancies, KS tumors are usually polyclonal, and the spindle cells are diploid and genetically stable (29). Four patterns of KS can be distinguished (79). Classic KS, which is most often identified in elder Mediterranean men, is a relatively nonaggressive disease, usually presenting with a limited number of lesions on the lower extremities. Endemic KS, which is seen mainly in sub-Saharan Africa, is more aggressive. Neither classic nor endemic KS is associated with HIV infection. Epidemic KS occurs in HIV-infected individuals, is an AIDS-defining disease, and is the most common tumor in AIDS patients. It often presents as a widely disseminated cutaneous disease that can also involve the oral mucosa and the viscera. Immunodeficiency promotes KS, and the fourth type, iatrogenic KS, is seen in solid organ transplant recipients and other immunosuppressed individuals. However, HIV may also have a more direct role in tumorigenesis, for instance, by Tat-mediated promotion of spindle cell proliferation or by the general upregulation of cytokines associated with HIV infection (3).

KSHV infection is considered to be the critical factor for the development of all KS forms for several reasons (29). First, viral DNA is always found in the spindle cells of the tumors, including those in HIV-negative individuals. Although a percentage of spindle cells in KS display lytic gene expression, most are latently infected, and the proportion of latently infected cells increases as the lesions advance. Second, KSHV infection precedes the onset of KS. Third, KSHV seroprevalence tends to vary directly with the incidence of HIVindependent KS. Fourth, odds ratios for KSHV infection in KS are often over 1000 in case/ control epidemiological studies (24). However, other cofactors must be required for tumorigenesis, since the prevalence of KSHV infection is much greater than the incidence of KS.

KSHV infection is also strongly associated with two relatively rare B cell neoplasias, multicentric Castleman's disease (MCD) and primary effusion lymphoma (PEL). MCD is a polyclonal B cell lymphoproliferative disease with dissemination to multiple lymph nodes and other lymphoid tissues. In AIDS patients, MCD is invariably associated with KSHV infection, whereas approximately half the cases in HIV-negative individuals are KSHV associated. PEL is a monoclonal B cell malignancy with rapid proliferation of cells in the pleural, peritoneal, and pericardial cavities. It usually occurs in AIDS patients or other immunosuppressed individuals and often is associated with both KSHV and EBV infections.

KSHV has been the subject of relatively little vaccine research compared with other human oncoviruses and herpesviruses. Perhaps because of the generally better track record of antivirals for herpesviruses, there has been substantially more activity in developing pharmacologic agents than vaccines against KSHV (80). KSHV might also be considered a second tier vaccine target. KSHV-associated KS does represent approximately $1 \%$ of cancers attributable to infections and is the leading cause of male cancers in some African countries (63). However, primary infection is usually asymptomatic, and the implementation of effective antiretroviral therapies to treat HIV infections has led to a dramatic reduction of KS in most developed countries (24). Efforts to prevent or treat HIV infection may therefore have largely superseded more direct efforts to interrupt KSHV infection. 
A second contributing factor that has limited vaccine research is the lack of an animal model for KSHV infection and/or KS induction. Gammaherpesviruses have been identified in nonhuman primates (85) and in mice. However, only the murine virus, HMV68, has been exploited in development of vaccines, including several interesting ones involving live attenuated HMV68 clones engineered to prevent the establishment of latency (39).

Approximately one-quarter of KSHV's 86 genes appear to be involved in immune regulation (2), and these elaborate immune evasion strategies must be considered. Successful development of vaccines for HSV, EBV, and other herpesviruses may, in the future, provide valuable information and incentives for the development of a KSHV vaccine. However, a sufficiently attractive investment case would have to be made to interest substantial corporate participation in KSHV vaccine development.

\section{EPSTEIN-BARR VIRUS}

Epstein-Barr virus (EBV) was the first herpesvirus shown to be oncogenic for humans and was initially identified in 1964 (25). Like KSHV, it is a gammaherpesvirus $(47,68)$.

However, the vast majority of adults have been infected with EBV. In developing countries, most individuals are infected as young children, whereas in the developed world, acute infection commonly occurs in adolescents and young adults. The virus is spread orally and infects epithelial cells and B cells. Acute EBV infection may be asymptomatic or cause infectious mononucleosis (IM), which is uncommon in young children but occurs in at least one-quarter of these infections in adolescents and young adults. In IM, the peripheral blood is dominated by an enormous polyclonal expansion of CD8 T cells, in response to a variety of EBV antigens, which helps to control the infection. However, the virus establishes latent B cell infection in virtually all hosts. Some latently infected B cells express the so-called viral latent proteins (the EBNA and the LMP proteins). However, the main reservoir of latently infected cells, which are probably derived from memory B cells in the oropharyngeal lymphoid tissues, predominantly expresses EBER1 and EBER2, which are noncoding RNAs. Lytic infection, which commonly occurs at low levels in the mouths of healthy carriers, is limited to epithelial cells, which may become infected from latently infected B cells. Healthy carriers usually have neutralizing antibodies against the envelope glycoprotein, gp350, as well as antibodies against the viral capsid antigen and EBNA-1, a nonstructural intracellular protein $(47,68)$. Some evidence suggests an association between EBV infection and multiple sclerosis.

Immunosuppression, typically in the setting of organ transplantation or HIV infection, often results in increased EBV loads and a risk of EBV-associated mouth lesions known as oral hairy leukoplakia and more serious EBV-associated diseases (50). Organ transplant recipients on immunosuppressive drugs may develop poorly controlled EBV infection through oral acquisition or, more frequently, from latently infected donor B cells within the graft. The infection often leads to post-transplant lymphoproliferative disease (PTLD), which ranges from a polyclonal condition that usually arises in the first year after transplantation, to frank monoclonal immunoblastic lymphoma after a longer interval. HIVinfected patients are also at high risk of developing B cell lymphomas, of which about $50 \%$ are EBV associated. As noted above, patients with PEL may be infected with EBV in 
addition to KSHV. Unexpectedly, immunocompromised individuals may also develop EBVassociated smooth muscle cell tumors, leiomyomas, and leiomyosarcomas.

EBV infection in nonimmunosuppressed patients has been implicated in a variety of malignancies, predominantly lymphomas and epidermal cell tumors (50). These include Burkitt's lymphoma (BL), Hodgkin's lymphoma (HL), T and NK lymphomas, nasopharyngeal carcinoma (NPC), and gastric carcinoma. For many of these tumor types, EBV accounts for only some of the tumors, although certain histologic types within a particular tumor are virtually always EBV associated (Table 1). EBV is found in a portion of sporadic and AIDS-associated BL, HL, and gastric adenocarcinomas. However, EBV is universally associated with endemic BL (which is the most common childhood tumor in equatorial Africa), with nasal lymphoma of T or NK cells and virus-associated hemophagocytic syndrome (VAHS), with nonkeratinizing (and possibly also with keratinizing) NPC, and with the UNCT (undifferentiated carcinoma of the nasopharyngeal type) type of gastric carcinoma. The latent period for most of these tumors is believed to be decades, with a shorter estimate for BL (3-8 years) as a notable exception. The fact that the EBV-associated tumors only develop in a small minority of infected individuals indicates that infection alone is not sufficient for tumor development, although it may be necessary. The limited geographic distribution of endemic BL and NPC (which is mainly clustered in Southeast Asia) suggests that environmental, and possibly genetic, factors are important. Immune escape is a common feature of these malignancies (61).

Various forms of latent (i.e., nonproductive) EBV infection have been described $(47,68)$. Their significance lies in their association with the various diseases linked to EBV infection. The latent forms are usually classified as latency 0 , which as noted above is limited to EBER expression; latency I, which expresses EBNA1; latency II, which expresses EBNA1 and LMP1-2; and latency III, which expresses EBNA1-6 and LMP1-2. All forms express EBER1 and EBER2, and latency I-III express the BARTs (BamH1A RNAs) with uncertain coding capacity. BL is associated with latency I expression, HD and NPC with latency II, and IM and PTLD with latency III.

There have been some attempts to develop a prophylactic vaccine against EBV, with the main goal of preventing IM (43). The generally poor track record of vaccines against herpesviruses may have limited these efforts. However, it is reasonable to believe that a vaccine that could prevent IM would also reduce the incidence of malignant disease attributable to EBV infection, as well as that of the other nonmalignant EBV-associated diseases. Although the induction of sterilizing immunity might be ideal, a reduction in EBV load and IM might be sufficient, as evidence suggests a relationship between viral dose and disease. There are two EBV genotypes, and a successful vaccine would need to protect against both of them. EBV can infect and cause disease in several nonhuman primate species, with most studies having been carried out in the cotton-top tamarin. A recently described partially humanized mouse model of EBV infection may also have potential for increased understanding of protective immunity and vaccine development (78). As noted above, MHV68, a mouse gammaherpesvirus that causes disease with similarities to EBV, has been used to evaluate gammaherpesvirus vaccines. 
Therapeutic EBV vaccine efforts have been concerned with the latency antigens expressed in malignant disease, but the recognition that the viral gp350 contains the main neutralization epitopes has focused attention on its potential to serve as the basis for a prophylactic vaccine. Preclinical studies have suggested that gp350 is immunogenic and can confer some protection, but it is uncertain whether this is attributable to neutralizing antibodies, to the cellular immune response to gp350, or to a combination thereof. A phase II trial of a recombinant gp350 vaccine adjuvanted with AS04 in seronegative subjects was well tolerated, induced seroconversion in all vaccinees, and produced data suggestive of partial protection against IM but not against infection (75). These results suggest that it may be desirable to include other antigens in the vaccine. Most frequently, peptides from latent viral proteins that induce EBV-specific CD8 T cells are studied, given that such cells contribute to clearance of infection in otherwise healthy individuals and therapeutically in PTLD patients (70). A phase I trial of such a peptide vaccine, from the EBNA3 protein, in seronegative individuals has been carried out (23). The oil-in-water adjuvanted vaccine was well tolerated and induced an immune response in the volunteers. Given the HLA diversity in people, the authors estimate that about 25 CD8 T cell epitopes would be needed to cover more than $90 \%$ of the population in the developed world. Some preliminary efforts are in progress to combine latent and lytic viral antigens in a single vaccine (54). It has also recently been shown that EBV virus-like particles (VLPs) that express a wide spectrum of structural viral proteins, including gp350, can be efficiently produced and induce proliferation of EBVspecific CD4 T cells $(1,18)$. The potential of VLPs as a vaccine has not yet been reported, but given the efficacy of the HBV and HPV VLP vaccines, the authors suggest this might be a vaccination strategy to consider for EBV. However, if immune reactivity against latent proteins proves necessary for strong protection, the VLPs will not contain them.

\section{HUMAN T CELL LEUKEMIA VIRUS TYPE 1}

Human T cell leukemia virus type 1 (HTLV1) was first identified in 1980 from a patient with cutaneous T cell leukemia (66). Epidemiologic studies in Japan and North and South America established the role of HTLV1 in the clinical entity first designated in Japan as adult $\mathrm{T}$ cell leukemia/lymphoma (ATL), a rapidly fatal disease $(35,82)$. HTLV infection is endemic to certain regions, including southern Japan, the Caribbean basin, Central and South America, and central Africa (51). It is estimated that 15-20 million people worldwide are chronic HTLV carriers. Viral transmission to infants occurs commonly through the milk of infected mothers, although there is also sexual and blood-borne transmission. As with HBV infection, HTLV infection during infancy is more likely to result in a chronic carrier state. HTLV infection is asymptomatic in most carriers, but they are estimated to have a 1$5 \%$ lifetime risk of developing ATL. Carriers may also develop HAM/TSP (HTLVassociated myelopathy/tropical spastic paraparesis), an uncommon chronic progressive neurological disease, or other rare HTLV-associated inflammatory disorders. The nonstructural Tax protein appears to be important for the induction of ATL, but neither Tax nor any other viral protein appears to be expressed in about 50\% of ATL cases. These ATL cases may represent a form of hit-and-run tumorigenesis, especially as proviral insertional mutagenesis does not appear to be a common mechanism. Most HAM/TSP patients have strong viral-specific CTL predominantly against Tax, which may contribute to the 
pathogenesis of the disease. Indeed, in asymptomatic carriers and HAM/TSP patients, the frequency of CD8 T cells is usually Tax > Pol > Eng > Gag (33). Rabbits, rats, and partially humanized transgenic mice have been used to study HTLV infection and the cellular and humoral immune parameters that may regulate it $(40,57)$.

There are fewer than 4000 cases annually of ATL and the other HTLV-associated diseases. This rate of serious disease makes it unlikely that there will be universal vaccination to prevent primary infection, even in endemic areas. In addition, a simple intervention, refraining from breastfeeding, can prevent most transmission events (34). Theoretically, there might be a place for vaccination of carriers, with the goal of preventing progression to ATL and HAM/TSP. The Tax protein would be the likely target, and there is some preclinical evidence for efficacy by a multiepitope Tax peptide vaccine $(40,57,81)$. However, the latent period for ATL development is usually decades, and with the possible exception of demonstrating a reduction in the level of provirus, it is not clear that a shortterm surrogate end point could be used to determine vaccine efficacy. This implies that the main use of a vaccine would be to treat ATL. However, the lack of viral gene expression in about half of ATL cases limits the theoretical value of this approach for these patients.

\section{CONCLUSION}

Vaccines against oncoviruses provide exceptional opportunities for cancer prevention, opportunities that, importantly, have the potential for implementation even in low-resource settings. However, the successful development and deployment of an oncovirus vaccine requires the convergence of several factors, including an unequivocal causal association of a virus with a cancer, sufficient public health implications in developed and/or emerging countries to attract commercial investment, and an effective vaccine candidate. These criteria have clearly been met for HBV and HPV. HCV and EBV meet the first two criteria, but vaccine candidates await validation in clinical trials. The fact that acute or short-term persistent infection by both viruses is associated with substantial morbidity enhances their attractiveness as prophylactic vaccine targets, especially as the typically long interval between oncovirus infection and cancer development means that the reduction in malignant disease may not been seen until many years after vaccine implementation. The investment case for KSHV vaccines is weaker, and they have received relatively less attention. It is doubtful that there is sufficient incentive to produce a commercial HTLV vaccine. The search for new cancer-associated viruses continues, propelled in part by the potential that their identification would have for cancer prevention. Interesting candidates, such as Merkel cell polyomavirus (MCPyV) (27) and xenotropic murine leukemia virus-related virus (XMRV) (84), have recently been identified. These viruses, and likely yet undiscovered human viruses, may also become candidates for vaccine development, provided the link to cancer is convincingly established and the associated morbidity from cancer and/or other serious virus-associated disease is sufficiently prevalent.

\section{Glossary}

HPV human papillomavirus 


\begin{tabular}{|c|c|}
\hline HBV & hepatitis B virus \\
\hline $\mathrm{HCV}$ & hepatitis $\mathrm{C}$ virus \\
\hline EBV & Epstein-Barr virus \\
\hline KSHV & Kaposi's sarcoma-associated herpesvirus \\
\hline HTLV & human $\mathrm{T}$ cell lymphotropic virus \\
\hline Prophylactic vaccine & $\begin{array}{l}\text { a vaccine that prevents a viral infection or the disease it } \\
\text { causes }\end{array}$ \\
\hline Oncovirus & a virus that is an etiologic agent in a cancer \\
\hline Therapeutic vaccine & $\begin{array}{l}\text { a vaccine that treats a viral infection or the disease induced } \\
\text { by the infection }\end{array}$ \\
\hline HCC & hepatocellular carcinoma \\
\hline Neutralization & $\begin{array}{l}\text { prevention of virus infection, usually in reference to an } \\
\text { antibody-dependent mechanism }\end{array}$ \\
\hline VLP & virus-like particle \\
\hline CIN 2+ & cervical intraepithelial neoplasia grade 2 or higher \\
\hline KS & Kaposi's sarcoma \\
\hline
\end{tabular}

\section{LITERATURE CITED}

1. Adhikary D, Behrends U, Feederle R, Delecluse HJ, Mautner J. Standardized and highly efficient expansion of Epstein-Barr virus-specific CD4+ T cells by using virus-like particles. J Virol. 2008; 82:3903-11. [PubMed: 18272580]

2. Areste C, Blackbourn DJ. Modulation of the immune system by Kaposi's sarcoma-associated herpesvirus. Trends Microbiol. 2009; 17:119-29. [PubMed: 19230674]

3. Barillari G, Ensoli B. Angiogenic effects of extracellular human immunodeficiency virus type 1 Tat protein and its role in the pathogenesis of AIDS-associated Kaposi's sarcoma. Clin Microbiol Rev. 2002; 15:310-26. [PubMed: 11932235]

4. Bartosch B, Dubuisson J, Cosset FL. Infectious hepatitis C virus pseudoparticles containing functional E1-E2 envelope protein complexes. J Exp Med. 2003; 197:633-42. [PubMed: 12615904]

5. Baseman JG, Koutsky LA. The epidemiology of human papillomavirus infections. J Clin Virol. 2005; 32(Suppl 1):S16-24. [PubMed: 15753008]

6. Beasley RP, Hwang LY, Lin CC, Chien CS. Hepatocellular carcinoma and hepatitis B virus. A prospective study of 22,707 men in Taiwan. Lancet. 1981; 2:1129-33. [PubMed: 6118576]

7. Bosch FX, Burchell AN, Schiffman M, Giuliano AR, de Sanjose S, et al. Epidemiology and natural history of human papillomavirus infections and type-specific implications in cervical neoplasia. Vaccine. 2008; 26(Suppl 10):K1-16. [PubMed: 18847553]

8. Bosch FX, Lorincz A, Munoz N, Meijer CJ, Shah KV. The causal relation between human papillomavirus and cervical cancer. J Clin Pathol. 2002; 55:244-65. [PubMed: 11919208]

9. Bosch FX, Ribes J, Cleries R, Diaz M. Epidemiology of hepatocellular carcinoma. Clin Liver Dis. 2005; 9:191-211. [PubMed: 15831268]

10. Brown DR, Kjaer SK, Sigurdsson K, Iversen OE, Hernandez-Avila M, et al. The impact of quadrivalent human papillomavirus (HPV; types 6, 11, 16, and 18) L1 virus-like particle vaccine 
on infection and disease due to oncogenic nonvaccine HPV types in generally HPV-naive women aged 16-26 years. J Infect Dis. 2009; 199:926-35. [PubMed: 19236279]

11. Cantarella G, Liniger M, Zuniga A, Schiller JT, Billeter M, et al. Recombinant measles virus-HPV vaccine candidates for prevention of cervical carcinoma. Vaccine. 2009; 27:3385-90. [PubMed: 19200837]

12. Chang MH, Chen CJ, Lai MS, Hsu HM, Wu TC, et al. Universal hepatitis B vaccination in Taiwan and the incidence of hepatocellular carcinoma in children. Taiwan Childhood Hepatoma Study Group. N Engl J Med. 1997; 336:1855-59. [PubMed: 9197213]

13. Chang MH, You SL, Chen CJ, Liu CJ, Lee CM, et al. Decreased incidence of hepatocellular carcinoma in hepatitis B vaccinees: a 20-year follow-up study. J Natl Cancer Inst. 2009; 101:134855. [PubMed: 19759364]

14. Chang Y, Cesarman E, Pessin MS, Lee F, Culpepper J, et al. Identification of herpesvirus-like DNA sequences in AIDS-associated Kaposi's sarcoma. Science. 1994; 266:1865-69. [PubMed: 7997879]

15. Chisari FV, Ferrari C. Hepatitis B virus immunopathogenesis. Annu Rev Immunol. 1995; 13:29_ 60. [PubMed: 7612225]

16. Chotiyaputta W, Lok AS. Hepatitis B virus variants. Nat Rev Gastroenterol Hepatol. 2009; 6:45362. [PubMed: 19581904]

17. David MP, Van Herck K, Hardt K, Tibaldi F, Dubin G, et al. Long-term persistence of anti-HPV-16 and -18 antibodies induced by vaccination with the AS04-adjuvanted cervical cancer vaccine: modeling of sustained antibody responses. Gynecol Oncol. 2009; 115:S1-6. [PubMed: 19217149]

18. Delecluse HJ, Feederle R, Behrends U, Mautner J. Contribution of viral recombinants to the study of the immune response against the Epstein-Barr virus. Semin Cancer Biol. 2008; 18:409-15. [PubMed: 18938248]

19. Demberg T, Robert-Guroff M. Mucosal immunity and protection against HIV/SIV infection: strategies and challenges for vaccine design. Int Rev Immunol. 2009; 28:20-48. [PubMed: 19241252]

20. Durst M, Gissmann L, Ikenberg H, zur Hausen H. A papillomavirus DNA from a cervical carcinoma and its prevalence in cancer biopsy samples from different geographic regions. Proc Natl Acad Sci USA. 1983; 80:3812-15. [PubMed: 6304740]

21. Dustin LB, Rice CM. Flying under the radar: the immunobiology of hepatitis C. Annu Rev Immunol. 2007; 25:71-99. [PubMed: 17067278]

22. Edmunds WJ, Medley GF, Nokes DJ, Hall AJ, Whittle HC. The influence of age on the development of the hepatitis B carrier state. Proc Biol Sci. 1993; 253:197-201. [PubMed: 8397416]

23. Elliott SL, Suhrbier A, Miles JJ, Lawrence G, Pye SJ, et al. Phase I trial of a CD8+ T-cell peptide epitope-based vaccine for infectious mononucleosis. J Virol. 2008; 82:1448-57. [PubMed: 18032491]

24. Engels EA, Biggar RJ, Hall HI, Cross H, Crutchfield A, et al. Cancer risk in people infected with human immunodeficiency virus in the United States. Int J Cancer. 2008; 123:187-94. [PubMed: 18435450]

25. Epstein MA, Achong BG, Barr YM. Virus particles in cultured lymphoblasts from Burkitt's lymphoma. Lancet. 1964; 1:702-3. [PubMed: 14107961]

26. Feinstone SM, Kapikian AZ, Purcell RH, Alter HJ, Holland PV. Transfusion-associated hepatitis not due to viral hepatitis type A or B. N Engl J Med. 1975; 292:767-70. [PubMed: 163436]

27. Feng H, Shuda M, Chang Y, Moore PS. Clonal integration of a polyomavirus in human Merkel cell carcinoma. Science. 2008; 319:1096-100. [PubMed: 18202256]

28. Fraillery D, Baud D, Pang SY, Schiller J, Bobst M, et al. Salmonella enterica serovar Typhi Ty21a expressing human papillomavirus type $16 \mathrm{~L} 1$ as a potential live vaccine against cervical cancer and typhoid fever. Clin Vaccine Immunol. 2007; 14:1285-95. [PubMed: 17687110]

29. Ganem D. KSHV infection and the pathogenesis of Kaposi's sarcoma. Annu Rev Pathol. 2006; 1:273-96. [PubMed: 18039116]

30. Ganem D. Kaposi's sarcoma-associated herpesvirus. 2007:2847-88. See Ref. 48. 
31. Gillison ML, Chaturvedi AK, Lowy DR. HPV prophylactic vaccines and the potential prevention of noncervical cancers in both men and women. Cancer. 2008; 113:3036-46. [PubMed: 18980286]

32. Goldstein ST, Zhou F, Hadler SC, Bell BP, Mast EE, Margolis HS. A mathematical model to estimate global hepatitis B disease burden and vaccination impact. Int J Epidemiol. 2005; 34:1329-39. [PubMed: 16249217]

33. Goon PK, Biancardi A, Fast N, Igakura T, Hanon E, et al. Human T cell lymphotropic virus (HTLV) type-1-specific CD8+ T cells: frequency and immunodominance hierarchy. J Infect Dis. 2004; 189:2294-98. [PubMed: 15181578]

34. Hino S, Katamine S, Miyata H, Tsuji Y, Yamabe T, Miyamoto T. Primary prevention of HTLV-1 in Japan. Leukemia. 1997; 11(Suppl 3):57-59. [PubMed: 9209297]

35. Hinuma Y, Nagata K, Hanaoka M, Nakai M, Matsumoto T, et al. Adult T-cell leukemia: antigen in an ATL cell line and detection of antibodies to the antigen in human sera. Proc Natl Acad Sci USA. 1981; 78:6476-80. [PubMed: 7031654]

36. Houghton M, Abrignani S. Prospects for a vaccine against the hepatitis C virus. Nature. 2005; 436:961-66. [PubMed: 16107836]

37. Howley PM, Lowy DR. Pappillomaviruses. 2007:2299-354. See Ref. 48.

38. Jagu S, Karanam B, Gambhira R, Chivukula SV, Chaganti RJ, et al. Concatenated multitype L2 fusion proteins as candidate prophylactic pan-human papillomavirus vaccines. J Natl Cancer Inst. 2009; 101:782-92. [PubMed: 19470949]

39. Jia Q, Freeman ML, Yager EJ, McHardy I, Tong L, et al. Induction of protective immunity against murine gammaherpesvirus 68 infection in the absence of viral latency. J Virol. 2010; 84:2453-65. [PubMed: 20015983]

40. Kannagi M, Harashima N, Kurihara K, Ohashi T, Utsunomiya A, et al. Tumor immunity against adult T-cell leukemia. Cancer Sci. 2005; 96:249-55. [PubMed: 15904464]

41. Kanodia S, Da Silva DM, Kast WM. Recent advances in strategies for immunotherapy of human papillomavirus-induced lesions. Int J Cancer. 2008; 122:247-59. [PubMed: 17973257]

42. Kato T, Date T, Miyamoto M, Furusaka A, Tokushige K, et al. Efficient replication of the genotype 2a hepatitis $\mathrm{C}$ virus subgenomic replicon. Gastroenterology. 2003; 125:1808-17. [PubMed: 14724833]

43. Khanna R, Moss DJ, Burrows SR. Vaccine strategies against Epstein-Barr virus-associated diseases: lessons from studies on cytotoxic T-cell-mediated immune regulation. Immunol Rev. 1999; 170:49-64. [PubMed: 10566141]

44. Kines RC, Thompson CD, Lowy DR, Schiller JT, Day PM. The initial steps leading to papillomavirus infection occur on the basement membrane prior to cell surface binding. Proc Natl Acad Sci USA. 2009; 106:20458-63. [PubMed: 19920181]

45. Kirnbauer R, Booy F, Cheng N, Lowy DR, Schiller JT. Papillomavirus L1 major capsid protein self-assembles into virus-like particles that are highly immunogenic. Proc Natl Acad Sci USA. 1992; 89:12180-84. [PubMed: 1334560]

46. Kjaer SK, Sigurdsson K, Iversen OE, Hernandez-Avila M, Wheeler CM, et al. A pooled analysis of continued prophylactic efficacy of quadrivalent human papillomavirus (types 6/11/16/18) vaccine against high-grade cervical and external genital lesions. Cancer Prev Res. 2009; 2:868-78.

47. Klein E, Kis LL, Klein G. Epstein-Barr virus infection in humans: from harmless to life endangering virus-lymphocyte interactions. Oncogene. 2007; 26:1297-305. [PubMed: 17322915]

48. Knipe DM, Howley PM, editorsField's Virology. Philadelphia: Lippincott Williams \& Wilkins; 2007.

49. Koutsky LA, Ault KA, Wheeler CM, Brown DR, Barr E, et al. A controlled trial of a human papillomavirus type 16 vaccine. N Engl J Med. 2002; 347:1645-51. [PubMed: 12444178]

50. Kutok JL, Wang F. Spectrum of Epstein-Barr virus-associated diseases. Annu Rev Pathol. 2006; 1:375-404. [PubMed: 18039120]

51. Latirmore MD, Franchini G. Human T-cell leukemia types 1 and 2. 2007:2071-105. See Ref. 48.

52. Levy-Bruhl D, Bousquet V, King LA, O'Flanagan D, Bacci S, et al. The current state of introduction of HPV vaccination into national immunization schedules in Europe: results of the VENICE 2008 survey. Eur J Cancer. 2009; 45:2709-13. [PubMed: 19695863] 
53. Liang TJ. Hepatitis B: the virus and disease. Hepatology. 2009; 49:S13-21. [PubMed: 19399811]

54. Lockey TD, Zhan X, Surman S, Sample CE, Hurwitz JL. Epstein-Barr virus vaccine development: a lytic and latent protein cocktail. Front Biosci. 2008; 13:5916-27. [PubMed: 18508632]

55. Lowy DR, Solomon D, Hildesheim A, Schiller JT, Schiffman M. Human papillomavirus infection and the primary and secondary prevention of cervical cancer. Cancer. 2008; 113:1980-93. [PubMed: 18798536]

56. Lupberger J, Hildt E. Hepatitis B virus-induced oncogenesis. World J Gastroenterol. 2007; 13:7481. [PubMed: 17206756]

57. Lynch MP, Kaumaya PT. Advances in HTLV-1 peptide vaccines and therapeutics. Curr Protein Pept Sci. 2006; 7:137-45. [PubMed: 16611139]

58. Mast EE, Ward JW. Hepatitis B vaccines. In: Plotkin SA, Orenstei WA, Offit PA, editors Vaccines. 5. Maryland Heights, MO: Saunders; 2004. 205-42.

59. Maynard JE. Passive immunization against hepatitis B: a review of recent studies and comment on current aspects of control. Am J Epidemiol. 1978; 107:77-86. [PubMed: 341692]

60. McAleer WJ, Buynak EB, Maigetter RZ, Wampler DE, Miller WJ, Hilleman MR. Human hepatitis B vaccine from recombinant yeast. Nature. 1984; 307:178-80. [PubMed: 6318124]

61. Munz C, Moormann A. Immune escape by Epstein-Barr virus associated malignancies. Semin Cancer Biol. 2008; 18:381-87. [PubMed: 18996483]

62. Paavonen J, Naud P, Salmeron J, Wheeler CM, Chow SN, et al. Efficacy of human papillomavirus (HPV)-16/18 AS04-adjuvanted vaccine against cervical infection and precancer caused by oncogenic HPV types (PATRICIA): final analysis of a double-blind, randomised study in young women. Lancet. 2009; 374:301-14. [PubMed: 19586656]

63. Parkin DM. The global health burden of infection-associated cancers in the year 2002. Int J Cancer. 2006; 118:3030-44. [PubMed: 16404738]

64. Pestka JM, Zeisel MB, Blaser E, Schurmann P, Bartosch B, et al. Rapid induction of virusneutralizing antibodies and viral clearance in a single-source outbreak of hepatitis C. Proc Natl Acad Sci USA. 2007; 104:6025-30. [PubMed: 17392433]

65. Pica F, Volpi A. Transmission of human herpesvirus 8: an update. Curr Opin Infect Dis. 2007; 20:152-56. [PubMed: 17496573]

66. Poiesz BJ, Ruscetti FW, Gazdar AF, Bunn PA, Minna JD, Gallo RC. Detection and isolation of type $\mathrm{C}$ retrovirus particles from fresh and cultured lymphocytes of a patient with cutaneous T-cell lymphoma. Proc Natl Acad Sci USA. 1980; 77:7415-19. [PubMed: 6261256]

67. Poovorawan Y, Chongsrisawat V, Theamboonlers A, Bock HL, Leyssen M, Jacquet JM. Persistence of antibodies and immune memory to hepatitis B vaccine 20 years after infant vaccination in Thailand. Vaccine. 2010; 28:730-36. [PubMed: 19892043]

68. Richardson AB, Kieff E. Epstein-Barr virus. 2007:2655-700. See Ref. 48.

69. Roberts JN, Buck CB, Thompson CD, Kines R, Bernardo M, et al. Genital transmission of HPV in a mouse model is potentiated by nonoxynol-9 and inhibited by carrageenan. Nat Med. 2007; 13:857-61. [PubMed: 17603495]

70. Savoldo B, Goss JA, Hammer MM, Zhang L, Lopez T, et al. Treatment of solid organ transplant recipients with autologous Epstein Barr virus-specific cytotoxic T lymphocytes (CTLs). Blood. 2006; 108:2942-49. [PubMed: 16835376]

71. Schiller JT, Castellsague X, Villa L, Hildesheim A. An update of prophylactic human papillomavirus L1 virus-like particle vaccine clinical trial results. Vaccine. 2008; 26S:K53-61.

72. Schiller JT, Lowy DR. Immunogenicity testing in human papillomavirus virus-like-particle vaccine trials. J Infect Dis. 2009; 200:166-71. [PubMed: 19519255]

73. Shefer A, Markowitz L, Deeks S, Tam T, Irwin K, et al. Early experience with human papillomavirus vaccine introduction in the United States, Canada and Australia. Vaccine. 2008; 26(Suppl 10):K68-75. [PubMed: 18847559]

74. Shepard CW, Finelli L, Alter MJ. Global epidemiology of hepatitis C virus infection. Lancet Infect Dis. 2005; 5:558-67. [PubMed: 16122679]

75. Sokal EM, Hoppenbrouwers K, Vandermeulen C, Moutschen M, Leonard P, et al. Recombinant gp350 vaccine for infectious mononucleosis: a phase 2, randomized, double-blind, placebo- 
controlled trial to evaluate the safety, immunogenicity, and efficacy of an Epstein-Barr virus vaccine in healthy young adults. J Infect Dis. 2007; 196:1749-53. [PubMed: 18190254]

76. Stamatatos L, Morris L, Burton DR, Mascola JR. Neutralizing antibodies generated during natural HIV-1 infection: good news for an HIV-1 vaccine? Nat Med. 2009; 15:866-70. [PubMed: 19525964]

77. Stoll-Keller F, Barth H, Fafi-Kremer S, Zeisel MB, Baumert TF. Development of hepatitis C virus vaccines: challenges and progress. Expert Rev Vaccines. 2009; 8:333-45. [PubMed: 19249975]

78. Strowig T, Gurer C, Ploss A, Liu YF, Arrey F, et al. Priming of protective T cell responses against virus-induced tumors in mice with human immune system components. J Exp Med. 2009; 206:1423-34. [PubMed: 19487422]

79. Sullivan RJ, Pantanowitz L, Casper C, Stebbing J, Dezube BJ. HIV/AIDS: epidemiology, pathophysiology, and treatment of Kaposi sarcoma-associated herpesvirus disease: Kaposi sarcoma, primary effusion lymphoma, and multicentric Castleman disease. Clin Infect Dis. 2008; 47:1209-15. [PubMed: 18808357]

80. Sullivan RJ, Pantanowitz L, Dezube BJ. Targeted therapy for Kaposi sarcoma. BioDrugs. 2009; 23:69-75. [PubMed: 19489649]

81. Sundaram R, Lynch MP, Rawale S, Dakappagari N, Young D, et al. Protective efficacy of multiepitope human leukocyte antigen-A*0201 restricted cytotoxic T-lymphocyte peptide construct against challenge with human T-cell lymphotropic virus type 1 Tax recombinant vaccinia virus. J Acquir Immune Defic Syndr. 2004; 37:1329-39. [PubMed: 15483462]

82. Tajima K. The fourth nation-wide study of adult T-cell leukemia/lymphoma (ATL) in Japan: estimates of risk of ATL and its geographical and clinical features. The T- and B-cell Malignancy Study Group. Int J Cancer. 1990; 45:237-43. [PubMed: 2303290]

83. Tang H, Grise H. Cellular and molecular biology of HCV infection and hepatitis. Clin Sci. 2009; 117:49-65. [PubMed: 19515018]

84. Urisman A, Molinaro RJ, Fischer N, Plummer SJ, Casey G, et al. Identification of a novel Gammaretrovirus in prostate tumors of patients homozygous for R462Q RNASEL variant. PLoS Pathog. 2006; 2:e25. [PubMed: 16609730]

85. Westmoreland SV, Mansfield KG. Comparative pathobiology of Kaposi sarcoma-associated herpesvirus and related primate rhadinoviruses. Comp Med. 2008; 58:31-42. [PubMed: 19793454]

86. Zanetti AR, Van Damme P, Shouval D. The global impact of vaccination against hepatitis B: a historical overview. Vaccine. 2008; 26:6266-73. [PubMed: 18848855]

87. Zhang P, Zhong L, Struble EB, Watanabe H, Kachko A, et al. Depletion of interfering antibodies in chronic hepatitis $\mathrm{C}$ patients and vaccinated chimpanzees reveals broad cross-genotype neutralizing activity. Proc Natl Acad Sci USA. 2009; 106:7537-41. [PubMed: 19380744] 


\section{SUMMARY POINTS}

1. Identification of a viral cause of a cancer provides an excellent opportunity to prevent the cancer by preventing or treating the associated viral infection.

2. Oncovirus vaccines are an intervention strategy with a reasonable chance of eventually reaching economically disadvantaged populations, where cancers associated with infectious disease are most prevalent.

3. As cancers induced by oncoviruses usually take many years to develop, the impetus for prophylactic vaccine development is greater if an oncovirus also causes substantial non-malignant disease soon after infection.

4. Universal delivery of the HBV prophylactic vaccine to infants has led to a significant decrease in chronic liver disease and HCC.

5. In clinical trials, the recently introduced prophylactic HPV vaccines were effective at preventing persistent infections and premalignant neoplasia caused by the HPV types targeted by the vaccines.

6. Efforts are underway to develop and test $\mathrm{HCV}$ and EBV vaccines. Effective vaccines would likely be commercially viable products.

7. Efforts to develop KSHV vaccines have been modest, in part because effective anti-HIV therapies have reduced the incidence of KSHV-associated cancers in developed countries.

8. There has been relatively little investment in prophylactic HTLV vaccines because the initial HTLV infection is asymptomatic and the cancers that it causes are relatively uncommon. 


\section{FUTURE ISSUES}

1. Can effective HCV, EBV, and KSHV vaccines be developed? Will they rely on the induction of neutralizing antibodies, as in the cases of HPV, HBV, and most other viral vaccines, or will $\mathrm{T}$ cell effector responses be needed?

2. How can uptake of the HBV and HPV vaccines be increased both in developed and developing countries?

3. Will second-generation HPV vaccines that might induce protection against more types and/or be delivered at low cost be successful in clinical trials? If so, will they eventually supplant the current HPV vaccines?

4. Will vaccines effective against prevalent infection be developed? If so, the long interval between infection and cancer would make oncoviruses excellent candidates for such vaccines.

5. Are there other infectious agents that cause a sufficient number of cancers and sufficient morbidity that they might be suitable candidates for vaccine development? 


\section{Table 1}

Virus-associated cancers worldwide ${ }^{a}$

\begin{tabular}{l|l|r|r}
\hline Virus & Cancer site & Attributable fraction $(\boldsymbol{\%})$ & Attributable cancers (×1000) \\
\hline HPV & Cervix & 100 & 492.8 \\
\hline HPV & Anus & 90 & 27.4 \\
\hline HPV & Vulva, vagina & 40 & 16.0 \\
\hline HPV & Penis & 40 & 10.5 \\
\hline HPV & Mouth & 3 & 8.2 \\
\hline HPV & Oropharynx & 12 & 6.3 \\
\hline HBV & Liver & 54 & 340.0 \\
\hline HCV & Liver & 31 & 195.0 \\
\hline EBV & Burkitt's lymphoma & 82 & 6.7 \\
\hline EBV & Hodgkin's lymphoma & 46 & 28.6 \\
\hline EBV & Nasopharyngeal carcinoma & 98 & 78.1 \\
\hline KSHV & Kaposi's sarcoma & 100 & 66.2 \\
\hline HTLV & Adult T cell leukemia/lymphoma & $2 b$ & 3.3 \\
\hline
\end{tabular}

${ }^{a}$ Adapted from Reference 63.

$b_{\text {Percent of all non-Hodgkin's lymphomas. }}$

Abbreviations: EBV, Epstein-Barr virus; HBV, hepatitis B virus; HCV, hepatitis C virus; HPV, human papillomavirus; HTLV, human T cell lymphotropic virus; KSHV, Kaposi's sarcoma-associated herpesvirus. 